\title{
Generalized Predictive Fuzzy Control of Coordinated Control System of Thermal Power Plant
}

\author{
Shihe $\mathrm{CHEN}^{1}$, Xi ZHANG ${ }^{1}$, Li ZHANG ${ }^{2}$, Jianguo DUAN ${ }^{2}$, Bingju \\ WANG $^{2}$, Yuguang NIU ${ }^{2}$, Zhongwei LIN ${ }^{2}$ \\ ${ }^{1}$ Electric Power Research Institute of Guangdong Power Grid Corporation, Guangzhou, 510000, \\ China \\ ${ }^{2}$ School of control and computer engineering, North China Electric Power University, Beijing, \\ 102206, China \\ email: hengfeng928@163.com
}

\begin{abstract}
Keywords: thermal power plants; coordinated control system; T-S fuzzy model; generalized predictive control
\end{abstract}

\begin{abstract}
In view of the nonlinearity and coupling in the coordinated control system (CCS) of thermal power plants, the paper introduces a Takagi-Sugeno (T-S) fuzzy model based generalized predictive control (GPC) of CCS. First, a T-S fuzzy model with three chosen typical operating point is employed to approximate the nonlinearity of the CCS of the thermal power plant. Then, based on the obtained T-S fuzzy model, a GPC controller is developed to achieve a desired performance. Finally, a living example of a 330MW thermal power unit is used to demonstrate GPC controller.
\end{abstract}

\section{Introduction}

Coordinated control system of thermal Power plant is a nonlinear, coupled and multivariable control object. The primary control objective of the CCS is to achieve a fast load response, while the deviation between process value (PV) and set point (SP) of the throttle pressure should be confined in an acceptable range [1]. At present, PID controllers are well accepted in the engineering field for their reliability and simplicity [2]. However, the parameters of the PID controllers are usually tuned with the approximately linearized model. In this situation, the dynamic control performance may not be guaranteed during the period of the frequent load response.

Model predictive control (MPC) is an effective control method, and it is more suitable to control industrial processes for horizon optimization and feedback correction. Generalized predictive control [3-5], one of MPC's, takes controlled auto-regressive integrated moving-average (CARIMA) model as the predictive model, however, an optimization performance based on actual demand is also chosen [6]. For the characteristics of self-correction, low requirement of the predictive model, and strong robustness, GPS has been deeply researched and widely used in the process control.

Linear system based GPC and its applications have been widely reported in the past. However, there are no efficient and systematic methods in the GPC design of nonlinear objects. T-S fuzzy modelling is an effective way to analysis and approximate the complex nonlinear systems [8], such as CCS of thermal power plant. In the consideration of this, it is nature to combine GPC and T-S fuzzy modeling in the control of nonlinear system. The T-S fuzzy model based GPC combines the characteristics of TS fuzzy modeling and GPC [9], where the T-S fuzzy modeling is employed to cope with the nonlinearity, and the GPC is developed to achieve a desired performance. Furthermore, the T-S fuzzy model is a linearized model essentially, and linear or nonlinear weighting method is used to obtain the final output. It allows that linear system based GPC can be easily extended into control of nonlinear system. [10].

This paper introduces a T-S fuzzy model based GPC of CCS of thermal power plant. First, a T-S fuzzy model with three chosen typical operating points is employed to approximate the nonlinearity of the CCS of the thermal power plan. Then, based on the obtained T-S fuzzy model, a GPC controller is developed to achieve a desired performance. Finally, a living example of a 300MW thermal power unit is used to demonstrate the contributions. 


\section{T-S FUZZY BASED GPC}

GPC is commonly based on the CARIMA model, which can be written as following form:

$$
\boldsymbol{A}\left(z^{-1}\right) \boldsymbol{y}(k)=\boldsymbol{B}\left(z^{-1}\right) \boldsymbol{u}(k-1)+\boldsymbol{C}\left(z^{-1}\right) \boldsymbol{\xi}(k) / \Delta
$$

Here $\boldsymbol{u}(k) \in R^{1 \times m}, \boldsymbol{\xi}(k) \in R^{1 \times n}$ and $\boldsymbol{y}(k) \in R^{1 \times n}$ denote manipulated vector, white noise vector and output vector, respectively. The details about those variables are given as follows:

$$
\left\{\begin{array}{llll}
\boldsymbol{y}(k)=\left[\begin{array}{lllll}
y_{1}(k) & y_{2}(k) & \cdots & y_{n}(k)
\end{array}\right]^{\mathrm{T}}, \boldsymbol{u}(k)=\left[\begin{array}{llll}
u_{1}(k) & u_{2}(k) & \cdots & u_{m}(k)
\end{array}\right]^{\mathrm{T}} \\
\boldsymbol{\xi}(k)=\left[\begin{array}{lllll}
\xi_{1}(k) & \xi_{2}(k) & \cdots & \xi_{n}(k)
\end{array}\right]^{\mathrm{T}}
\end{array}\right.
$$

Where $\Delta=1-z^{-1}$ is the difference operator, matrixes $\boldsymbol{A}\left(z^{-1}\right) \in R^{\mathrm{n} \times \mathrm{n}}, \boldsymbol{B}\left(\mathrm{z}^{-1}\right) \in R^{n \times m}$ and $\boldsymbol{C}\left(\mathrm{z}^{-1}\right) \in R^{n \times n}$ are:

$$
\left\{\begin{array}{l}
\boldsymbol{A}\left(z^{-1}\right)=\boldsymbol{I}_{n \times n}+\boldsymbol{A}_{1} z^{-1}+\boldsymbol{A}_{2} z^{-2}+\cdots+\boldsymbol{A}_{n_{a}} z^{-n_{a}}, \boldsymbol{B}\left(z^{-1}\right)=\boldsymbol{B}_{0}+\boldsymbol{B}_{1} z^{-1}+\boldsymbol{B}_{2} z^{-2}+\cdots+\boldsymbol{B}_{n_{b}} z^{-n_{b}} \\
\boldsymbol{C}\left(z^{-1}\right)=\boldsymbol{I}_{n \times n}+\boldsymbol{C}_{1} z^{-1}+\boldsymbol{C}_{2} z^{-2}+\cdots+\boldsymbol{C}_{n_{c}} z^{-n_{c}}
\end{array}\right.
$$

Assumption $\boldsymbol{C}\left(z^{-1}\right)=\boldsymbol{I}_{n \times n}$, the Diophantine equation is written as follows:

$$
\begin{gathered}
\boldsymbol{I}_{n \times n}=\boldsymbol{E}_{P}\left(z^{-1}\right) \boldsymbol{A}\left(z^{-1}\right) \Delta+z^{-j} \boldsymbol{F}_{P}\left(z^{-1}\right) \\
\boldsymbol{E}_{P}\left(z^{-1}\right) \boldsymbol{B}\left(z^{-1}\right)=\boldsymbol{G}_{P}\left(z^{-1}\right)+z^{-j} \boldsymbol{H}_{P}\left(z^{-1}\right)
\end{gathered}
$$

Where $\boldsymbol{E}_{P}\left(z^{-1}\right), \boldsymbol{F}_{P}\left(z^{-1}\right), \boldsymbol{G}_{P}\left(z^{-1}\right)$, and $\boldsymbol{H}_{P}\left(z^{-1}\right)$ are polynomial matrixes, which are determined by $\boldsymbol{A}\left(z^{-1}\right), \boldsymbol{B}\left(z^{-1}\right)$ and predictive horizon $P$.

$$
\left\{\begin{array}{l}
\boldsymbol{G}_{P}\left(z^{-1}\right)=\boldsymbol{G}_{0}+\boldsymbol{G}_{1} z^{-1}+\cdots+\boldsymbol{G}_{P-1} z^{-(P-1)}, \boldsymbol{H}_{P}\left(z^{-1}\right)=\boldsymbol{H}_{P, 0}+\boldsymbol{H}_{P, 1} z^{-1}+\cdots+\boldsymbol{H}_{P, n_{b}-1} z^{-\left(n_{b}-1\right)} \\
\boldsymbol{E}_{P}\left(z^{-1}\right)=\boldsymbol{E}_{0}+\boldsymbol{E}_{1} z^{-1}+\cdots+\boldsymbol{E}_{P-1} z^{-(P-1)}, \boldsymbol{F}_{P}\left(z^{-1}\right)=\boldsymbol{F}_{P, 0}+\boldsymbol{F}_{P, 1} z^{-1}+\cdots+\boldsymbol{F}_{P, n_{a}} z^{-n_{a}}
\end{array}\right.
$$

Here, all the coefficient matrices in $G_{P}\left(\mathrm{z}^{-1}\right), H_{P}\left(\mathrm{z}^{-1}\right)$ are $n \times m$ matrices, and all the coefficient matrices in $E_{p}\left(\mathrm{z}^{-1}\right), F_{p}\left(\mathrm{z}^{-1}\right)$ are $n \times n$ matrices.

Multiplying $z_{P} \boldsymbol{E}_{P}\left(\mathrm{z}^{-1}\right) \Delta$ into (1). By using (2) and (3), the preditive output at $P$-th setp $\boldsymbol{y}(k+P \mid k)$ can be obtained. Then, the predictive vector from $y(k+1 \mid k)$ to $y(k+P \mid k)$ can be rewrite as:

$$
\hat{\boldsymbol{Y}}=\boldsymbol{G} \Delta \boldsymbol{U}+\boldsymbol{F} \boldsymbol{y}(k)+\boldsymbol{H} \Delta \boldsymbol{u}(k-1)+\boldsymbol{E}
$$

Where $P$ and $M$ are prediction horizon and control horizon, respectively, and

$$
\begin{aligned}
\hat{\boldsymbol{Y}} & =\left[\hat{\boldsymbol{y}}^{\mathrm{T}}(k+1 \mid k), \hat{\boldsymbol{y}}^{\mathrm{T}}(k+2 \mid k), \cdots, \hat{\boldsymbol{y}}^{\mathrm{T}}(k+P \mid k)\right]^{\mathrm{T}}, \Delta \boldsymbol{U}=\left[\Delta \boldsymbol{u}^{\mathrm{T}}(k), \Delta \boldsymbol{u}^{\mathrm{T}}(k+2), \cdots, \Delta \boldsymbol{u}^{\mathrm{T}}(k+M-1)\right]^{\mathrm{T}} \\
\boldsymbol{F} & =\left[\boldsymbol{F}_{1}^{\mathrm{T}}\left(z^{-1}\right), \boldsymbol{F}_{2}^{\mathrm{T}}\left(z^{-1}\right), \cdots, \boldsymbol{F}_{n}^{\mathrm{T}}\left(z^{-1}\right)\right]^{\mathrm{T}}, \boldsymbol{H}=\left[\boldsymbol{H}_{1}^{\mathrm{T}}\left(z^{-1}\right), \boldsymbol{H}_{2}^{\mathrm{T}}\left(z^{-1}\right), \cdots, \boldsymbol{H}_{n}^{\mathrm{T}}\left(z^{-1}\right)\right]^{\mathrm{T}} \\
\boldsymbol{E} & =\left[\boldsymbol{\xi}^{\mathrm{T}}(k+1) \boldsymbol{E}_{1}^{\mathrm{T}}\left(z^{-1}\right), \boldsymbol{\xi}^{\mathrm{T}}(k+2) \boldsymbol{E}_{2}^{\mathrm{T}}\left(z^{-1}\right), \cdots, \boldsymbol{\xi}^{\mathrm{T}}(k+n) \boldsymbol{E}_{n}^{\mathrm{T}}\left(z^{-1}\right)\right]^{\mathrm{T}} \\
\boldsymbol{G} & =\left[\begin{array}{cccc}
\boldsymbol{G}_{0} & & \\
\boldsymbol{G}_{1} & \boldsymbol{G}_{0} & \\
\vdots & \vdots & \ddots & \\
\boldsymbol{G}_{m-1} & \boldsymbol{G}_{m-2} & \cdots & \boldsymbol{G}_{0} \\
\vdots & \vdots & \vdots & \vdots \\
\boldsymbol{G}_{n-1} & \boldsymbol{G}_{n-2} & \cdots & \boldsymbol{G}_{n-m}
\end{array}\right]
\end{aligned}
$$

Ignoring the influence of $\boldsymbol{E}$ on the unknown noise, the predictive output of the system in (4) can be rewritten as follows:

$$
\hat{\boldsymbol{Y}}=\boldsymbol{G} \Delta \boldsymbol{U}+\boldsymbol{F} \boldsymbol{y}(k)+\boldsymbol{H} \Delta \boldsymbol{u}(k-1)
$$

The T-S fuzzy method is widely used to approximate nonlinear systems [11]. T-S fuzzy model can be represented by using if-then structures.

Plant rule $i$ :

If $\quad x_{1}$ is $M^{i}$ and $\cdots$ and $x_{m}$ is $M_{m}^{i}$.

Then $y^{i}=b^{i}{ }_{0}+b^{i}{ }_{1} x_{1}+\cdots+b_{m}^{i} x_{m}$ for $i=1,2 \cdots c$

The final output of the fuzzy system is inferred as follows: 


$$
y=\sum_{i=1}^{c} \mu_{i} y^{i} / \sum_{i=1}^{c} \mu_{i}, \mu_{i}=\prod_{j=1}^{m} M_{j}^{i}\left(x_{j}\right)
$$

Where $c$ is number of If-Then rules, $x_{1}, x_{2}, x_{m}$ are premise variables, $y^{i}$ is output of the $i$-th T-S fuzzy rule, $M_{1}^{i}, M_{2}^{i} \cdots M_{m}^{i}$ are fuzzy sets, $b_{j}^{i}(j=1,2 \cdots m)$ is conclusion parameters which can be finded in the reference [12].

For combining with GPC, in this paper it is assumed that:

$$
T_{i}=y^{i}=\boldsymbol{G}_{i} \Delta \boldsymbol{U}_{i}+\boldsymbol{F}_{i} \boldsymbol{y}(k)+\boldsymbol{H}_{i} \Delta \boldsymbol{u}(k-1)
$$

While, the output of the whole fuzzy model can be written as follows:

$$
\hat{\boldsymbol{y}}=\sum_{i=1}^{c} K_{i} T_{i} / \sum_{i=1}^{c} K_{i}
$$

$K_{i}$ represents the weight ratio of the output of the subsystem model, the expression is:

$$
K_{i}=\mu_{i} / \sum_{i=1}^{c} \mu_{i}
$$

Where $\mu_{i}(i=1,2, \cdots, c)$. represents the membership degree.

The predicted output can be gotten by substituting (7) into (8):

$$
\hat{\boldsymbol{y}}=\sum_{i=1}^{c} K_{i}\left[\boldsymbol{G}_{i} \Delta \boldsymbol{U}_{i}+\boldsymbol{F}_{i} \boldsymbol{y}(k)+\boldsymbol{H}_{i} \Delta \boldsymbol{u}(k-1)\right] / \sum_{i=1}^{c} K_{i}
$$

The performance index function of the T-S fuzzy GPC in the form of a vector is chosen as follows:

$$
J=(\boldsymbol{Y}-\boldsymbol{W})^{\mathrm{T}}(\boldsymbol{Y}-\boldsymbol{W})+\Delta \boldsymbol{U}^{\mathrm{T}} \boldsymbol{\lambda} \Delta \boldsymbol{U}
$$

Where

$$
\begin{aligned}
& \boldsymbol{W}=\left[\begin{array}{ll}
\boldsymbol{w}^{\mathrm{T}}(k+1) & \boldsymbol{w}^{\mathrm{T}}(k+2) \cdots \boldsymbol{w}^{\mathrm{T}}(k+P)
\end{array}\right]^{\mathrm{T}} \\
& \boldsymbol{w}(k)=\left[\begin{array}{ll}
w_{1}(k) & w_{2}(k) \cdots w_{n}(k)
\end{array}\right]^{\mathrm{T}} \\
& \lambda=\operatorname{diag}\left(\lambda_{i}\right) \quad \lambda_{i}=\operatorname{diag}\left(\lambda_{i s}\right)
\end{aligned}
$$

Here, $\boldsymbol{Y}$ is the predictive output of the output variable, $\boldsymbol{W}$ is the corresponding expected value, $\lambda_{\text {is }}$ are control weighting parameters.

With (10), the performance in (11) can be rewritten as:

$$
J=(\boldsymbol{S} \Delta \boldsymbol{U}+\boldsymbol{R}-\boldsymbol{W})^{\mathrm{T}}(\boldsymbol{S} \Delta \boldsymbol{U}+\boldsymbol{R}-\boldsymbol{W})+\Delta \boldsymbol{U}^{\mathrm{T}} \boldsymbol{\lambda} \Delta \boldsymbol{U}
$$

Where

$$
\left\{\boldsymbol{S}=\sum_{i=1}^{c} K_{i} \boldsymbol{G}_{i} / \sum_{i=1}^{c} K_{i}, \boldsymbol{R}=\sum_{i=1}^{c} K_{i}\left[\boldsymbol{F}_{\boldsymbol{i}} y(k)+\boldsymbol{H}_{i} \Delta u(k-1)\right] / \sum_{i=1}^{c} K_{i}\right.
$$

Taking Order $\frac{\partial J}{\partial \Delta U}=0$, the optimization problem can be solved analytically for $\Delta U$.

$$
\Delta \boldsymbol{U}=\left(\boldsymbol{S}^{\mathrm{T}} \boldsymbol{S}+\lambda\right)^{-1} \boldsymbol{G}^{\mathrm{T}}(\boldsymbol{W}-\boldsymbol{R})
$$

Then, the output of the controller is

$$
\Delta \boldsymbol{u}(k)=\left[\begin{array}{llll}
I & 0 & \cdots & 0
\end{array}\right] \Delta \boldsymbol{U}
$$

\section{Simulation Research}

\section{Simplified nonlinear model of CCS}

The simplified model of a 330MW subcritical thermal power unit is given as following:

$$
\begin{aligned}
& r_{\mathrm{m}}^{\prime}=e^{-18 s} u_{\mathrm{b}}, \quad 120 \frac{d r_{0}}{d t}=-r_{0}+r_{\mathrm{m}}^{\prime}, \quad 3266 \frac{d P_{\mathrm{b}}}{d t}=-0.2501 P_{\mathrm{t}} u_{\mathrm{t}}+6.77 r_{0} \\
& 12 \frac{d N}{d t}=-N+0.2501 P_{\mathrm{t}} u_{\mathrm{t}}, \quad P_{\mathrm{t}}=P_{\mathrm{b}}-0.0000455\left(P_{\mathrm{t}} u_{\mathrm{t}}\right)^{1.3}
\end{aligned}
$$


Where, ub and ut which are input signals, denote the fired coal and the opening of turbine governing valve, respectively. Pt is throttle pressure and $\mathrm{N}$ is the output active power. The Pt and $\mathrm{N}$ are output variables. The detailed information can be found in [13].

The CCS is a double-input double-output system, which can be divided into two double-input single-output systems. In the boiler follow $(\mathrm{BF})$ mode, $u_{\mathrm{t}}$ is used to control the output active power to trach the instruments from the automatic dispatch system (ADS), while the dynamic of $P_{\mathrm{t}}$ is maintained by $u_{\mathrm{b}}$. The dynamic of the steam turbine is much faster in comparison with the boiler. In this situation, the disturbance from $u_{\mathrm{b}}$ can be quickly eliminated by manipulating the $u_{\mathrm{t}}$ in the active power loop. However, for the large inertia of the boiler, the disturbance from opening or closing the main steam valve, will cause the oscillations of $P_{t}$, which makes a large deviation between the set point (SP) and process values (PV) of $P_{\mathrm{t}}$. So that, the key technique in the BF model is to maintain the deviations of $P_{\mathrm{t}}$ in an acceptable range. Based on this consideration, the PID controller for the output active power is adopted to manipulate the $u_{\mathrm{t}}$. While, the T-S fuzzy based GPC is used to control the boiler by manipulating $u_{\mathrm{b}}$. The introduced schematic of CCS is shown in Fig. 1.

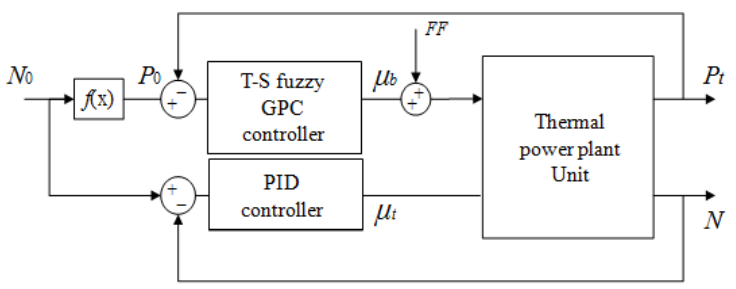

Figure 1. T-S fuzzy GPC structure of the coordinated control system of power plant

\section{Linearized Model of CCS}

The T-S fuzzy method is based on the linear model, thus, the nonlinear model in (12) should be linearized firstly. The linearized model at the chosen operating point can be obtained by use small signal linearization method [14-15]. In this paper, three linearized models based on the three typical operating points, which are chosen at $50 \%, 70 \%$, and $90 \%$ of the rated capacity of thermal power unit, are used to construct the T-S fuzzy model of the CCS. The specific parameters are defined as shown in table 1.

\begin{tabular}{cccc} 
TABLE I. & \multicolumn{4}{c}{ PARAMETERS OF THE BALANCE OPERATING POINT } \\
\hline No. & $\mathbf{1}$ & $\mathbf{2}$ & $\mathbf{3}$ \\
Operating point & $\mathbf{9 0} \%$ & $\mathbf{7 0 \%}$ & $\mathbf{5 0 \%}$ \\
\hline Load N/MW & 297 & 231 & 165 \\
\hline Pressure $\mathbf{P} / \mathbf{M P}$ & 17.27 & 15.5 & 12.17 \\
\hline
\end{tabular}

The linearized model of CCS can be rewritten as following form.

$$
\left[\begin{array}{l}
\Delta P_{\mathrm{t}} \\
\Delta N
\end{array}\right]=T(s) G_{0}(s) B(s)\left[\begin{array}{l}
\Delta u_{\mathrm{b}} \\
\Delta u_{\mathrm{t}}
\end{array}\right]
$$

Where $T(s)$ is used to describe the acting process of steam turbine, $B(s)$ is used to describe the dynamic process of fuel, and the core model of boiler-turbine coordinated system can be represented as $G_{0}(\mathrm{~s})$.

The CARIMA model of boiler-side, which is double-input single-output, can be obtained by discretizing the system in (13). With the fuzzy sets and membership function shown in Fig. 2, the predictive fuzzy CARIMA model of the boiler-side in the CCS can be constructed by using those three chosen operating points.

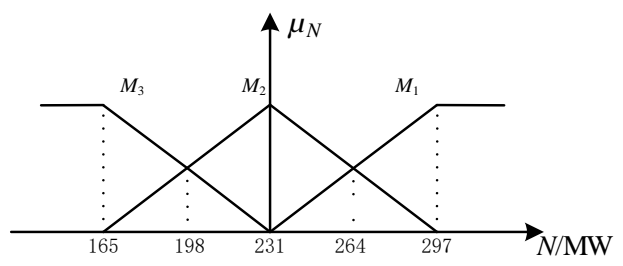

Figure 2. The membership function of power 


\section{Dynamic simulation}

In order to show the performance of the proposed T-S fuzzy based GPC in the thermal power plant, the simulations are performed in the MATLAB/SIMULATION. The control parameters of the GPC controller are sampling period $T s=1 \mathrm{~s}$, the prediction horizon $P=75$, the control horizon $M=3$, and the control weighting parameter $\lambda=0.4$. It is known that the sawtooth waves of ADS instruction are the most difficult for CCS to maintain acceptable performance. In this paper, the load responses with different rates of sawtooth waves are analyzed, such as $6 \mathrm{MW} / \mathrm{min}$ and $8 \mathrm{MW} / \mathrm{min}$.

Case 1: The load responses at rate of $6 \mathrm{MW} / \mathrm{min}$ with different controllers, such as the proposed GPC schematic and direct instruction balance (DIB) [16] are displayed in Fig. 3 and Fig. 4 respectively, where subfigure (a) and (c) show the overall response, subfigure (b) and (d) show the detailed enlarged curve of subfigure (a) and (c) respectively. The DIB schematic is based on the conventional PID controller, of which output is calculated by the deviation between the PV and SP. This infers that the DIB has little effective capability to cope with the objects with large inertia, long time delay, especially for the shape of ADS instrument as sawtooth waves. The evidence can be found in Fig. 3 (a) and Fig. 4 (a). From the Fig. 3 (b) and Fig. 4 (b), it is seen that the deviation between the SP and PV of throttle pressure $P_{\mathrm{t}}$ is larger when DIB is used. The output active power is also affected by the oscillation of $P_{t}$, where the evidence can be found in Fig. 3 (a) and Fig. 4 (a).

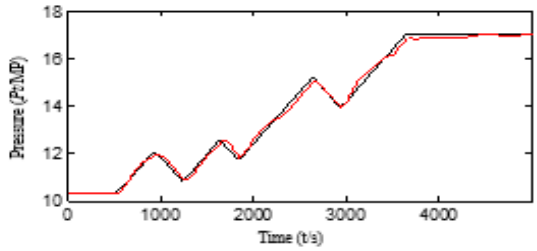

(a)

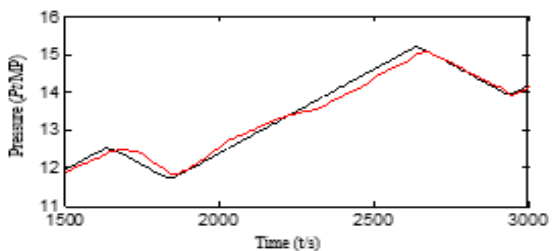

(b).

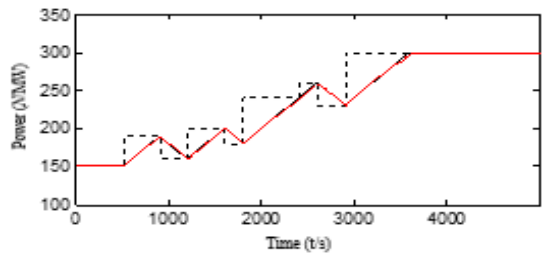

(c).

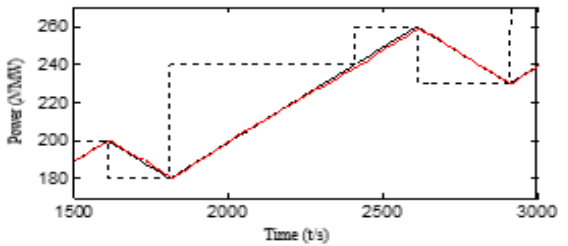

(d).

Set value —— Process value - ADS ---

Figure 3. The response of CCS with the propsoed T-S fuzzy GPC schemaic at 6MW/min

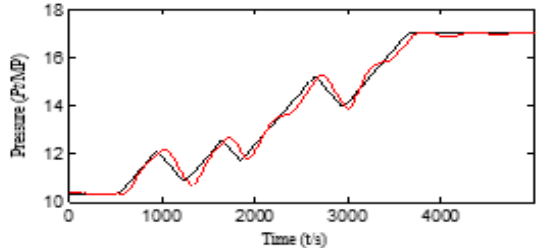

(a)

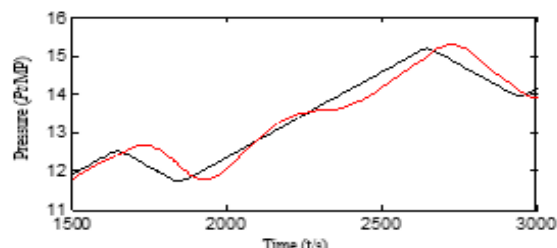

(b).:

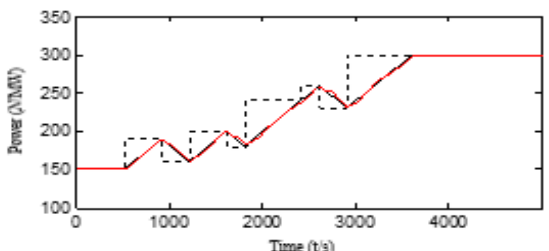

(c).

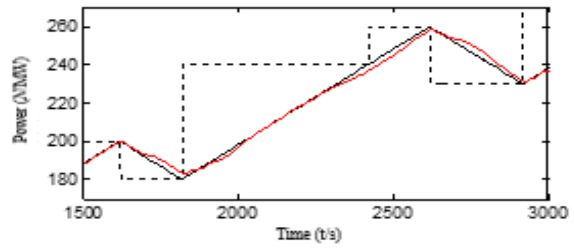

(d).

Set value - $\quad$ Process value - ADS ---

Figure 4. The response CCS of with the DIB schematic at $6 \mathrm{MW} / \mathrm{min}$

Case 2: In this case, the load rate is $8 \mathrm{MW} / \mathrm{min}$, which is larger than case 1 used rate. The larger rate needs a fast response for both boiler and turbine side controllers. The responses of CCS with proposed GPC and conventional DIB schematics are displayed in Fig. 5 and Fig. 6, respectively, where the subfigures have the same meaning as the Case 1 used. In comparison with the Case 1 , is can be found that the CCS performance with the DIB schematic is worsen under such 


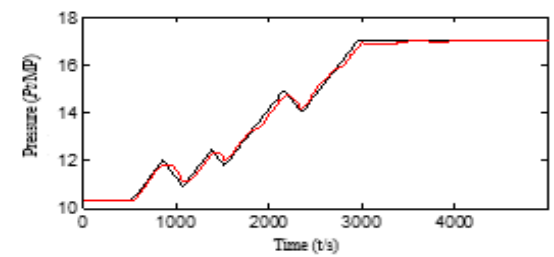

(a).

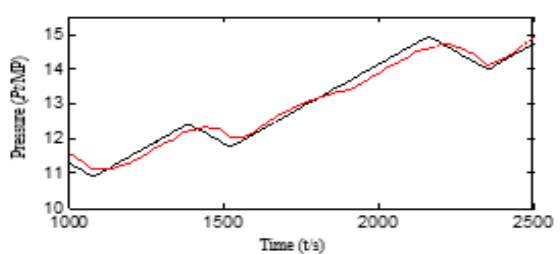

(b).

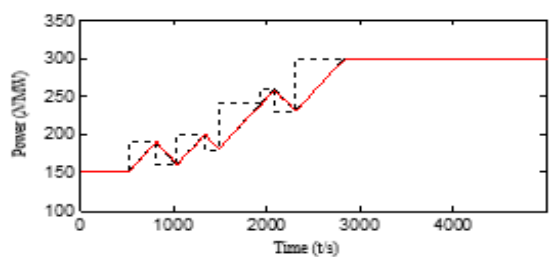

(c).

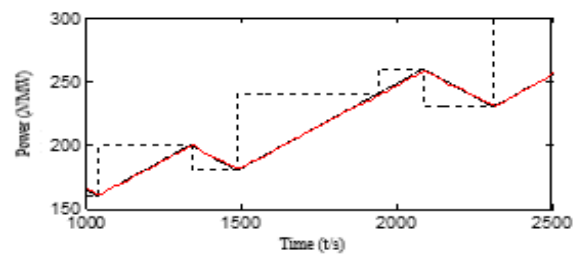

(d).

Set value —_ Process value - ADS ----

Figure 5. T-S fuzzy GPC control curves under $80 \%$ power change rate

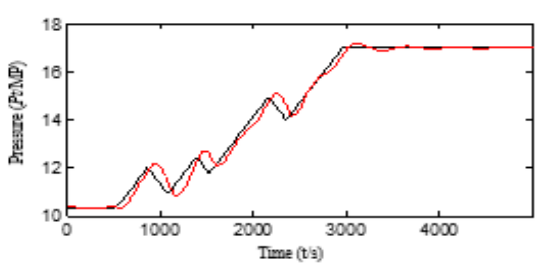

(a)

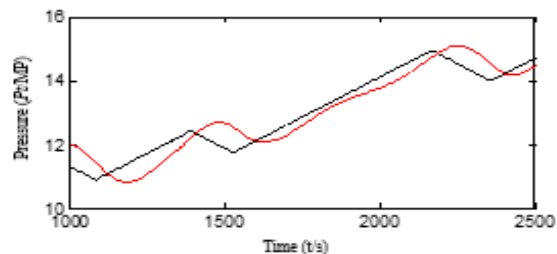

(b).

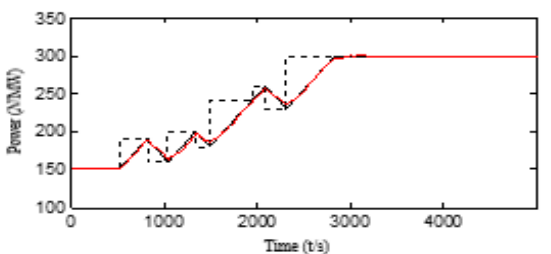

(c).

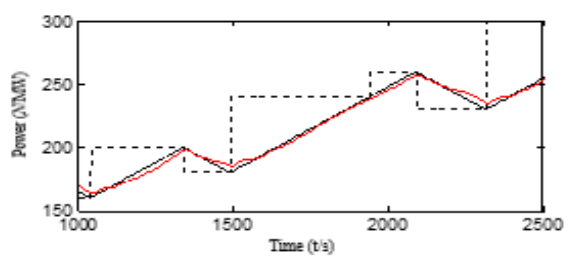

(d).

Set value —_ Process value - ADS ----

Figure 6. PID control curves under $80 \%$ power change rate

a large rate. However, the CCS with the proposed GPC schematic maintains a satisfied performance. The evidences can be found in Fig. 5 (a) and (c).

The simulations under different rates show that the proposed fuzzy based GPC controller provides a desired performance of the throttle pressure of thermal power plant, which benefits to a fast load response significantly.

\section{Conclusion}

This paper introduces a T-S fuzzy based GPC control of CCS of thermal power plant. The T-S fuzzy method is employed to approximate the nonlinear boiler-turbine system. Then, based on the obtained T-S fuzzy model, the GPC is developed to control the throttle pressure by manipulating the total amount of fired coal. The simulations show the contributions of the GPC controller in the performance of the CCS.

This paper demonstrates that the CCS performance can be improved by using a suitable advanced control schematic, such as T-S fuzzy control and GPC. Consequently, the frequency stability can be improved by the improvement of CCS.

\section{Acknowledgement}

This work is supported by the National Key Basic Research Program of China (973 Program) under Grant No.2012CB215203, and by the National Nature Science Foundation of China under Grant No.61203043. 


\section{References}

[1] HU Lingjun, WANG Wenlan, FENG Yongxiang. Study of the generalized perdictive control for generating plant in heat power plant [J]. Control theory and applications, 2003,05:5-7+14.[2] CAO Yongjun. The research of neural network generalized predictive control for thermal power plant [D]. Huhehaote: Inner Mongolia University of Technology,2006:2.

[3] LI Qi’an, LI Ping, LI Yue.Input and output constrained adaptive generalized predictive control for diagonal CARIMA model [J]. Chinese Journal of Scientific Instrument, 2008,29(7):1483-1488.

[4] DING Baocang. Predictive control theory and methods [M]. Beijing: China Machine Press,2008:54-63.

[5] CHEN Zhiwang, LIU Wenlong, LIU Zhihui. Fast algorithm for Toeplitz transformation of inverse matrix in generalized predictive control [J], Chinese Journal of Scientific Instrument, 2010,31(7):1626-1631.

[6] LI Guoyong, YANG Lijuan. Neural-Fuzzy-Predictive control and their Matlab implementation [M]. third ed. Beijing: Publishing House of Electronics Industry, 2013,05:291-292(in Chinese)

[7] HUO Weijing. A PID generalized predictive control of a kind of nonlinear system [D]. Qinhuangdao: Yanshan University, 2009: 1-2.

[8] XIE Fei. T-S fuzzy model identification study [D]. Nanjing: Southeast University, 2008. DOI:10.7666/d.y1386786.

[9] Takigi T, Sugeno M. Fuzzy identification of system and its application to modeling and control [J]. IEEE Transactions on System Man Cybernet, 1985, Vol.15(1):16-32.

[10] WANG Shubin, SHAN Shengnan, LUO Xionglin. Nonlinear predictive control based on T-S fuzzy model and particle-swarm optimization [J]. CIESC Journal, 2012,S1:176-187.

[11] WANG Wei, LI Xiaoli. Multiple model adaptive control [M]. Beijing: Science Press.2001:102-106.

[12] Takagi T, Sugeno M. Fuzzy identification of system and its application to modeling and control [J]. IEEE Trans on Systems, Man, and Cybernetic, 1985,15(1):116-132.

[13] TIAN Liang, ZENG Deliang, LIU Xinping etal. A simplified nonlinear dynamic model of 500MW plant [J]. Power Engineering, 2004,04:522-525.

[14] ZHENG Yafeng, CHEN Yanqiao, WEI Pingbao etal. Research on coordinated control system of 330MW plant based on fuzzy multiple model [J]. Proceedings of the CSU-EPSA, 2006,18(2): 71-74, 104. DOI: 10.3969 /j.issn.1003-8930.2006.02.016.

[15] TIAN Liang. Research of plant plant nonlinearity dynamic model [D]. North China Electric Power University (Hebei),2005.45-47.

[16] Zhao Songlie, Wang Wang. Optimization of DIB control strategy in 600MW Unit [J]. Zhejiang Electric Power,2006,03:42-45. 